\title{
Disponibilite, Valeurs Marchande Et Nutritionnelle Des Sous-Produits Agricoles Et Agroindustriels Utilises Dans L'alimentation Des Ruminants Au Benin
}

\author{
Montcho $M$. \\ Laboratoire de Zootechnie (LZ), Ecole des Sciences et Techniques de \\ Production Animale (ESTPA), Faculté des Sciences Agronomiques (FSA), \\ Université d’Abomey Calavi (UAC), Recette principale, Cotonou, Bénin \\ Babatoundé S. \\ LZ/ESTPA/FSA/UAC, 01 BP 526 Recette principale, Cotonou, Bénin \\ Aboh B. A. \\ Ecole d'Aquaculture de la Vallée, Université d’Agriculture de Kétou, Bénin \\ Bahini M. J. D. \\ LZ/ESTPA/FSA/UAC, 01 BP 526 Recette principale, Cotonou, Bénin \\ Chrysostome A. A. M. C. \\ Laboratoire de Recherche Avicole et de Zoo-Économie, \\ Zootechnie et Amélioration Génétique des Animaux, FSA/UAC, \\ Recette principale, Cotonou, Bénin

\section{Mensah G. A.} \\ Centre de Recherches Agricoles d'Agonkanmey, \\ Institut National des Recherches Agricoles du bénin (INRAB), \\ Recette principale, Cotonou, Bénin
}

doi: 10.19044/esj.2016.v12n33p422 URL:http://dx.doi.org/10.19044/esj.2016.v12n33p422

\begin{abstract}
The availabilities and the commercial values of 31 agricultural and agro-industrial by-products were evaluated through a semi structural interview carried out on 360 breeders of Benin. A sample of each diet was collected to determine its chemical composition and nutritive values. Maize bran, cassava peels, cakes of soya and cotton are available in any season and have a commercial value. Among fruit by-products used in the food of the ruminants, the pineapple peels occupy the first place and their commercial values are three times higher than those of the cassava peels. Soya cakes has a very high commercial value (higher than $200 \mathrm{FCFA} / \mathrm{kg}$ ).The leguminous pods and the cereal hulls are also largely available but without commercial value. Roots cassava and tubers of yam by-products and the cakes are very digestible and these values do not go down below $60 \%$. Low values of
\end{abstract}


digestibility were observed on hulls and stems of cereals and leguminous pods. These digestibilities are generally below $40 \%$. The groundnut haulms are more digestible than cowpea haulms (57.8 vs $49.7 \%$ ). Cotton cakes and palm oil cakes are less digestible than the other oil cakes. The digestibilities of crude protein (DCP) are very low in fruit and cereals by-products. Except for the pods of leguminous, all the leguminous by-products provided considerable quantities in DCP. The studies give opportunities of choice of food supplementation to ruminants during the dry season where graminaceous and the other herbaceous becoming rare in the natural pasture.

Keywords: By-products, availability, commercial value, nutritive values, Benin

\section{Résumé}

Les disponibilités et les valeurs marchandes de trente et un (31) sousproduits agricoles et agroindustriels ont été déterminées à travers une interview semi structurée réalisée auprès de trois cent soixante (360) éleveurs du Bénin. Par la suite, un échantillon de chaque aliment a été prélevé pour déterminer sa composition chimique et ses valeurs nutritives. Le son de maïs, les épluchures de manioc, les tourteaux de soja et de coton étaient disponibles en toutes saisons avec une valeur marchande. Parmi les sousproduits de fruits valorisés dans l'alimentation des ruminants, les pelures d'ananas occupaient la première place et leurs valeurs marchandes étaient de trois fois supérieures à celles des épluchures de manioc. Les tourteaux de soja aussi bien valorisés en alimentation des monogastriques non herbivores que des ruminants avaient une valeur marchande très élevée (supérieure à 200 FCFA le kg). Les gousses de légumineuses et les coques de céréales étaient aussi largement disponibles, mais sans valeur marchande. Les sousproduits de racines de manioc et tubercules d'igname ainsi que les tourteaux étaient très digestibles et ces valeurs de digestibilité de la matière organique (dMO) ne descendaient pas en-dessous de 60 \%. Les plus faibles valeurs de digestibilité ont été observées au niveau des coques et tiges de céréales ainsi que sur les gousses de légumineuses. Ces digestibilités étaient généralement en dessous de $40 \%$. Les fanes d'arachide ont été plus digestibles que les fanes de niébé ( $\mathrm{dMO}=57,8$ contre 49,7 \%). Les tourteaux de palmiste et de coton étaient moins digestibles que les autres tourteaux. Les sous-produits de céréales et de fruits se caractérisaient par leurs extrêmes pauvretés en matières azotées digestibles (MAD). En dehors des gousses, tous les autres sous-produits de légumineuses avaient des teneurs considérables en MAD. Ces résultats offrent des possibilités de choix de compléments alimentaires aux ruminants pendant la saison sèche où les graminées et autres herbacées fourragères deviennent rares dans les parcours naturels. 
Mots clés : Sous-produits, disponibilité, valeur marchande, valeur nutritive, Benin

\section{Introduction}

Au Bénin, l’élevage reste marqué par la prédominance des pratiques traditionnelles. Les effectifs des animaux sont estimés à 2.058 .000 bovins, 825.000 ovins, 1.640 .000 caprins, 383.000 porcins et 17087000 volailles en 2010 (DE, 2012). Les quantités produites de viandes et d’abats (56 300 tonnes), de lait (96 millions de litres) et des œufs (9.000 tonnes) issues de ces effectifs ne garantissent pas une couverture complète des besoins en protéines animales de la population. Ces productions sont encore insuffisantes au regard de l'accroissement démographique et de l'élévation du niveau de vie de la population béninoise. La production de viande pour satisfaire une demande sans cesse croissante en produits carnés constitue de ce fait une priorité nationale. Afin de réduire le déficit en protéines animales des populations, les importations de viandes et abats comestibles qui étaient de 2590 tonnes en 1996 sont passées à 31929 tonnes en 2005 (Babatoundé, 2005). D'après les statistiques de l'INSAE (2015), la tendance d'augmentation de ces importations est de 6,9 \% par an. L'un des principaux freins au développement du secteur de l'élevage est l'alimentation du troupeau, notamment en saison sèche. Les fourrages des parcours naturels qui constituent l'essentiel de l'alimentation des animaux herbivores sont quantitativement et qualitativement affectés par le rythme pluviométrique et l'évolution de la saison (Sinsin, 1993). Afin de maintenir le niveau de performance des animaux au cours de la saison déficitaire et de satisfaire la demande sans cesse croissante de la population en viande, il s'avère nécessaire d'avoir une meilleure connaissance des ressources alimentaires localement disponibles.

$\mathrm{Au}$ Bénin, le secteur agricole et agro-alimentaire génèrent de nombreux sous-produits. Ceux utilisés en élevage ont été évalués à 11. 043 tonnes (DE, 2012). Leur valorisation optimale peut à la fois améliorer les performances zootechniques des animaux et réduire les coûts alimentaires (CIRAD, 2009). L’un ou l'autre des éleveurs sollicités pour les incorporer dans les rations de leurs animaux manquent d'informations fiables afin de les utiliser dans des conditions optimales. Les informations concernant ces sousproduits sont fragmentaires, disparates, voire inexistantes pour certains. Pourtant le nombre de sous-produits ne cesse d'augmenter en rapport avec l'activité grandissante de la production agricole et de transformation artisanale réalisée par la population. En réalité, il n’existe pas de travaux qui recensent les sous-produits par zone agro écologique, évaluent leurs disponibilités et leurs valeurs marchandes et les consignent dans un même 
document. Ces connaissances sont pourtant fondamentales pour l'intensification et l'amélioration de la production animale au Bénin. En effet, la valeur marchande peut être définie à des fins économiques, scientifiques, sociologiques, culturelles, cultuelles, anthropologiques ou de développement durable.

\section{Matériels et Méthodes Milieu d'étude}

Les données ont été collectées dans cinq zones agro écologiques sur les huit que compte le Bénin (Figure 1). Il s'agit de la zone cotonnière du Nord-Bénin (Zone II). Le climat régnant est de type Nord-Soudanien caractérisé par une saison de pluie allant de mai à octobre et une saison sèche de novembre à avril. La température oscille entre 18 et $38^{\circ} \mathrm{C}$. C'est une zone de culture de sorgho, de maïs d'igname et de coton. La zone III dénommée zone des cultures vivrières du Sud-Borgou a une situation climatique identique à celle de la zone II. En plus des cultures vivrières précitées, se cultive l'anacardier. Dans ces deux zones, les quantités d'eau enregistrées étaient inférieures à $700 \mathrm{mn}$. La zone $\mathrm{V}$ des céréales ou encore zone cotonnière du Centre-Bénin est une zone de forte production de tubercules. Les légumineuses et le coton sont produits deux fois au cours de l'année. Les principales cultures produites sont le maïs, l'arachide, le manioc, l'igname, le soja, le coton et les cultures maraîchères. La zone VI est caractérisée par un climat de type soudano-guinéen avec deux saisons de pluies d'une pluviométrie moyenne de 800 à $1.400 \mathrm{~mm}$ par an. Les principales cultures de cette zone sont le maïs, le manioc, le niébé, l'ananas et le palmier à huile (INSAE, 2012).

Par ailleurs, la zone VII de par sa situation, se trouve dans la zone climatique de type subéquatoriale caractérisée par la succession annuelle de quatre saisons par alternance : deux saisons sèches (une grande, allant de décembre à mi-mars et une petite allant de mi-juillet à août) et deux saisons de pluies (une grande, allant de mi-mars à mi-juillet et une petite allant de septembre à novembre). Le niveau moyen des précipitations est de 1. 100 pour la grande saison et $800 \mathrm{~mm}$ pour la petite saison. Les températures moyennes mensuelles varient entre 27 et $31^{\circ} \mathrm{C}$. La base du système de production agricole est constituée du maïs associé au manioc, au niébé, à la tomate, au piment et les fruits tels que l'ananas, l'orange et la banane (INSAE, 2012). 


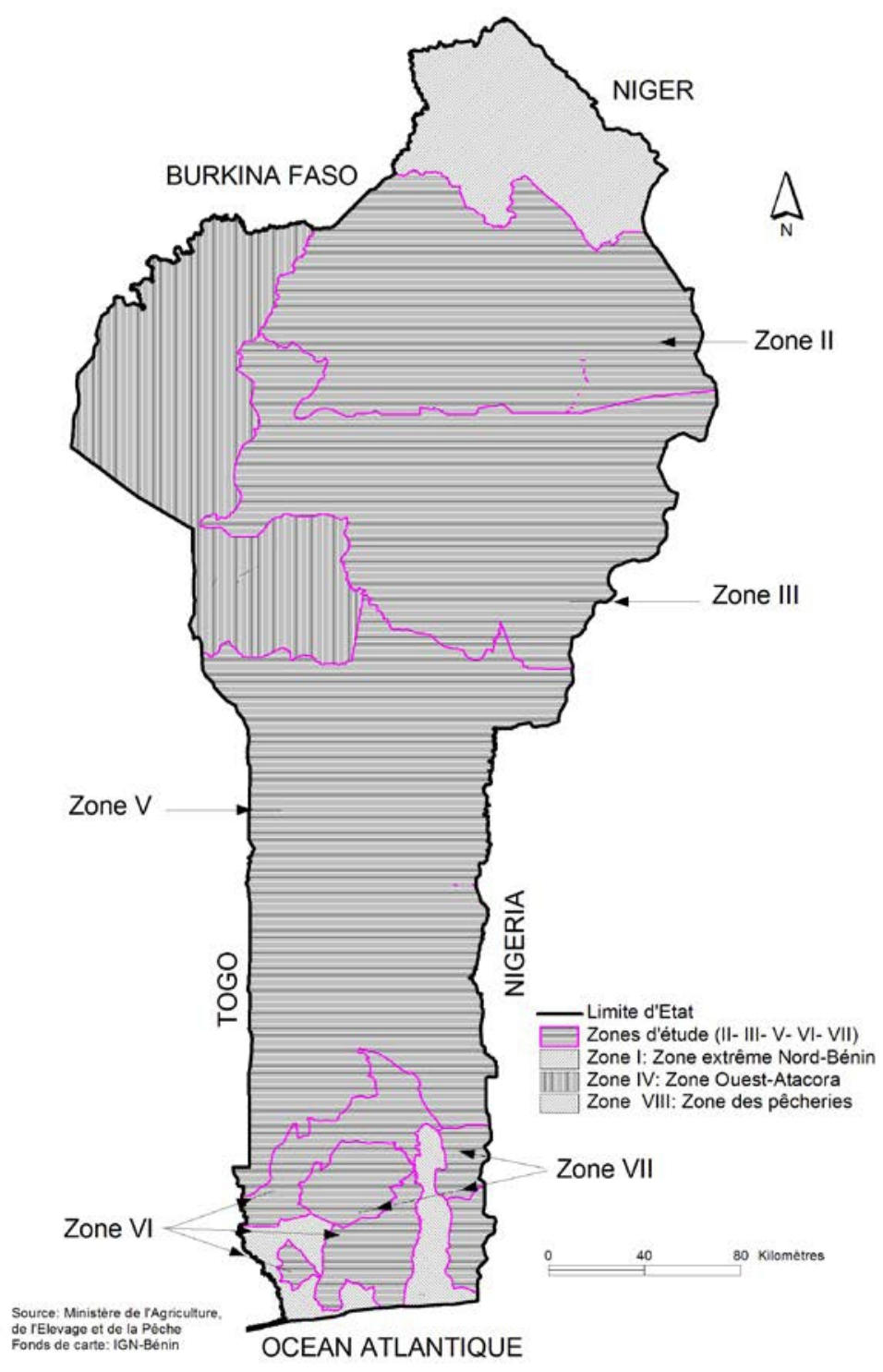

Figure 1 : Localisation des zones agro écologiques étudiées au Bénin.

\section{Collecte des données}

Une interview semi structurée a été utilisée pour la collecte des données. Le nombre d'éleveurs enquêtés était de 360. Ce nombre a été déterminé en utilisant l'approximation de la loi binomiale de Dagnelie (1986). Les données collectées lors des entretiens ont été relatives aux caractéristiques socio-économiques de l'élevage des ruminants et à 
l'utilisation des sous-produits agricoles et agro-industriels. Il s'agissait plus précisément de l’âge, du sexe, du niveau d'alphabétisation en langue locale, du niveau d’éducation formelle, de la principale activité professionnelle, des ressources alimentaires utilisées et de l'expérience dans l’élevage. Les données relatives à l'utilisation des sous-produits étaient constituées des types de sous-produits utilisés, de la disponibilité et des coûts d'acquisition de ces sous-produits. La présente étude a concerné au total neuf communes du Bénin (Calavi, Glazoué, Toffo, Allada, Dassa, Savalou, Nikki, Gogounou, Péhunco). Par commune, quatre villages ont été choisis avec l'appui des Responsables du Développement Rural (RDR). Le choix de ces villages a été fait suivant quatre principaux critères à savoir : l'existence des activités agricoles, de transformation des produits agricoles, la présence d'un effectif important de ruminants et l'accessibilité de la zone en toutes saisons.

\section{Détermination de la composition chimique}

Au total, 140 échantillons de sous-produits ont été recensés et soumis aux analyses chimiques de laboratoire. La matière sèche (MS, méthode ID 934,01), la matière organique (MO, méthode ID 942,05) et les matières azotées totales (MAT, méthode ID 954,01) ont été déterminées selon les méthodes officielles approuvées par AOAC (2000). Les teneurs en constituants pariétaux en particulier la cellulose brute (CB), Neutral et Acid Detergent Fiber (NDF et ADF) ont été déterminées selon la technique des sachets filtrants établie par ANKOM (ANKOM 200 Analyzer Fibre ; SKU: A200 et nb sp A200I). Par la suite les échantillons de sous-produits ont été soumis aux études de fermentescibilité in vitro en présence de jus de rumen de mouton et du tampon de Menke et al. (1979). Les échantillons de sousproduits (250 mg de MS) ont été incubés en seringues. L’incubation a été réalisée dans des seringues en verre de $100 \mathrm{ml}$ graduées par pas de $1 \mathrm{ml}$. Chaque seringue était équipée d'un robinet verrouillable à trois voies. Les pistons ont été lubrifiés à la vaseline avant chaque incubation. Les mesures de fermentescibilité en seringues ont été toutes répétées au cours d'une seconde période et a permis de prendre en compte les déviations associées à des facteurs non contrôlables, tels que la variabilité du jus de rumen et les facteurs environnementaux. A chaque période d'essai, trois répétitions d'un même échantillon de sous-produits ont été systématiquement effectuées. La position du piston était lue aux heures 0, 2, 4, 6, 8, 12, 24, 48, 72, 96, 120 et 144. Les volumes de gaz moyens produits ont été ajustés au modèle mathématique de France et al. (2000) :

$\mathbf{Y}=\mathrm{A}\left[1-\mathrm{e}^{[-\mathrm{b}(\mathrm{t}-\mathrm{T})-\mathrm{c}(\sqrt{ } \mathrm{t}-\sqrt{ } \mathrm{T})]}\right]$ si $\mathrm{t} \geq \mathrm{T}$ et

$\mathbf{Y}=0$ si $0<\mathrm{t}<\mathrm{T}$, avec

$\mathrm{Y}$ est le volume cumulé de gaz au temps $\mathrm{t}(\mathrm{ml})$; $\mathrm{t}$ le temps d'incubation (h); T le temps de latence (h); A (ml/g MS) la production 


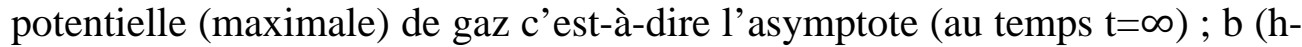
1) et c (h-1/2) des constantes.

L’équation du modèle établit une courbe cinétique théorique à partir des valeurs observées de volume de gaz. Le calcul a été réalisé par itérations successives jusqu’à la minimisation de la somme des carrés des écarts résiduels. Les paramètres issus de la modélisation ont été les volumes théoriques.

En utilisant les équations de régression de Menke et Steingass (1988), le volume de gaz mesuré après 24 heures (V24h, en ml/200 mg de MS) a été associé aux MAT et CT (g/kg MS) pour estimer la digestibilité in vivo de la matière organique (dMO) et de l'énergie métabolisable (EM) contenue dans les sous-produits.

Les valeurs énergétiques nettes (UFL, UFV) ont été estimées dans le système français (INRA, 1988). Les matières azotées digestibles (MAD) ont été calculées à partir de l'équation de régression établie par Jarrige et Demarquilly (1981) pour les fourrages (MAD =0,929MAT -3,52) et celle préconisée par Rivière (1991) pour les aliments concentrés (MAD = 0,916 MAT - 2,76).

\section{Analyses statistiques}

La statistique descriptive en termes de moyenne, minimum, maximum et de coefficient de variation a été utilisée sur les données de composition chimique et les valeurs nutritives des sous-produits. Par la suite, ces données ont été soumises à une analyse de variance (ANOVA) à un seul effet (catégorie de sous-produits) en utilisant la procédure du modèle linéaire général (PROC GLM) du logiciel SAS version 8.02 (SAS inc., 2008). Au terme de l'analyse, les différences entre les valeurs moyennes des sousproduits ont été comparées au moyen du test de Newman et Keuls (Dagnelie, 1986).

\section{Résultats}

Disponibilité et valeur marchande des sous-produits

Les sous-produits recensés par zone agro écologique ont été présentés dans le tableau 1. Quelle que soit la zone agro écologique, le son de maïs, les épluchures de manioc et le tourteau de soja étaient disponibles en toutes saisons et avaient une valeur marchande. Les pelures de fruits n'étaient disponibles que dans les zones des terres de barre et de dépression. Parmi les sous-produits de fruits valorisés dans l'alimentation des ruminants domestiques au Bénin, les pelures d'ananas occupaient la première place. Leur valeur marchande était de trois fois supérieure à celle des épluchures de manioc. Par contre, les pelures de banane, d'orange, de papaye et de fruit de Artocarpus Altilis (arbre à pain) appelé "bléfoutou" en langue locale 
«Fongbé » ont été très peu valorisées et n’avaient pas encore de valeur marchande. Dans la catégorie des sous-produits de céréales, les drèches artisanales de sorgho encore appelées drêches de" tchoukoutou "étaient disponibles dans presque toutes les zones agro écologiques et étaient relativement moins coûteuses. Les tourteaux avaient tous de valeur marchande élevée. Les tourteaux de soja aussi bien valorisé en alimentation des monogastriques non herbivores que des ruminants avaient une valeur marchande très élevée (généralement supérieure à 200 FCFA/kg). Les gousses de légumineuses et les coques de céréales très valorisés en alimentation des ruminants dans les pays sahéliens étaient aussi largement disponibles quelle que soit la saison. Le constat a été que ces sous-produits étaient sans valeur marchande.

Tableau 1. Disponibilité et valeur marchande des sous-produits inventoriés par zone agro écologique

\begin{tabular}{|c|c|c|c|c|c|}
\hline \multirow[b]{2}{*}{$\begin{array}{l}\text { Zones agro } \\
\text { écologiques }\end{array}$} & \multirow[b]{2}{*}{ Sous-produits } & \multicolumn{2}{|c|}{ Disponibilité } & \multicolumn{2}{|c|}{ Valeur Marchande } \\
\hline & & $\begin{array}{l}\text { Saison } \\
\text { sèche }\end{array}$ & $\begin{array}{c}\text { Saison } \\
\text { pluvieuse }\end{array}$ & $\begin{array}{c}\text { Valeur } \\
\text { Marchande }\end{array}$ & Prix d'achat \\
\hline Zone II : & Tourteaux de coton & +++ & +++ & Oui & 200 FCFA $/ \mathrm{kg}$ \\
\hline Zone cotonnière du & Tourteaux de soja & +++ & +++ & Oui & $250 \mathrm{FCFA} / \mathrm{kg}$ \\
\hline Nord-Bénin & Son de maïs & +++ & +++ & Oui & 25 FCFA / kg \\
\hline \multirow[t]{10}{*}{ (Gogounou) } & Drèches de sorgho (drêches de & +++ & +++ & Non & 40 FCFA / kg \\
\hline & tchoukoutou) & ++ & + & Non & - \\
\hline & Gousses de néré & ++ & ++ & Non & - \\
\hline & Gousses de soja & ++ & ++ & Oui & 50 FCFA / kg \\
\hline & Poudre de néré & +++ & ++ & Non & - \\
\hline & Coques de sorgho & +++ & ++ & Oui & $20 \mathrm{FCFA} / \mathrm{kg}$ \\
\hline & Tiges de sorgho & ++ & +++ & Non & - \\
\hline & Epluchures d'igname & ++ & +++ & Oui & $10 \mathrm{FCFA} / \mathrm{kg}$ \\
\hline & Epluchures de manioc & + & + & Non & - \\
\hline & Cabosses de cacao & & & & \\
\hline Zone III : & Tourteaux de karité & +++ & +++ & Oui & $120 \mathrm{FCFA} / \mathrm{kg}$ \\
\hline Zone vivrière du & Tourteaux de coton & +++ & +++ & Oui & $200 \mathrm{FCFA} / \mathrm{kg}$ \\
\hline Sud- & Son de maïs & +++ & +++ & Oui & $25 \mathrm{FCFA} / \mathrm{kg}$ \\
\hline Borgou(Ouassa- & Epluchures de manioc & +++ & +++ & Oui & $10 \mathrm{FCFA} / \mathrm{kg}$ \\
\hline \multirow[t]{11}{*}{ Pehunco ; Nikki ) } & Epluchures d’igname & +++ & +++ & Non & - \\
\hline & Drêches de sorgho (drêches de & +++ & +++ & Oui & $30 \mathrm{FCFA} / \mathrm{kg}$ \\
\hline & tchoukoutou) & +++ & ++ & Oui & $20 \mathrm{FCFA} / \mathrm{kg}$ \\
\hline & Tiges de sorgho; & +++ & ++ & Non & - \\
\hline & Gousses de néré; & +++ & + & Oui & $50 \mathrm{FCFA} / \mathrm{kg}$ \\
\hline & Fanes de niébé; & ++ & +++ & Oui & $300 \mathrm{FCFA} / \mathrm{kg}$ \\
\hline & Soja concassé; & +++ & +++ & Oui & $250 \mathrm{FCFA} / \mathrm{kg}$ \\
\hline & Tourteaux de soja; & +++ & ++ & Non & - \\
\hline & Gousses de soja; & +++ & ++ & Oui & $40 \mathrm{FCFA} / \mathrm{kg}$ \\
\hline & Fanes d'arachide & ++ & ++ & Oui & $50 \mathrm{FCFA} / \mathrm{kg}$ \\
\hline & Poudre de néré; & & & & \\
\hline Zone V: & Epluchures de manioc & +++ & +++ & Oui & $10 \mathrm{~F} \mathrm{FCFA} / \mathrm{kg}$ \\
\hline Zone cotonnière du & Remoulages de riz & +++ & +++ & Oui & $50 \mathrm{FCFA} / \mathrm{kg}$ \\
\hline Centre- Bénin & Son de niébé; & +++ & +++ & Oui & $75 F C F A / \mathrm{kg}$ \\
\hline (Glazoué-Savalou- & Tourteaux de palmiste & +++ & ++ & Oui & $150 \mathrm{FCFA} / \mathrm{kg}$ \\
\hline Dassa) & Son de riz; & +++ & +++ & Oui & $50 \mathrm{FCFA} / \mathrm{kg}$ \\
\hline & Epluchures de patate & +++ & ++ & Non & - \\
\hline & Tourteaux de soja & +++ & +++ & Non & $250 \mathrm{FCFA} / \mathrm{kg}$ \\
\hline & Remoulage de manioc (Garigo) & +++ & +++ & Oui & 175 FCFA/ kg \\
\hline & Son de riz & +++ & +++ & Oui & $50 \mathrm{FCFA} / \mathrm{kg}$ \\
\hline & Drèches de sorgho (drêches de & +++ & +++ & Oui & $45 \mathrm{FCFA} / \mathrm{kg}$ \\
\hline
\end{tabular}




\begin{tabular}{|c|c|c|c|c|c|}
\hline & $\begin{array}{c}\text { tchoukoutou) } \\
\text { Tourteaux d'arachide } \\
\text { Fanes d'arachide } \\
\text { Son de maïs }\end{array}$ & $\begin{array}{l}+++ \\
+++ \\
+++\end{array}$ & $\begin{array}{c}+++ \\
++ \\
+++\end{array}$ & $\begin{array}{l}\text { Oui } \\
\text { Oui } \\
\text { Oui }\end{array}$ & $\begin{array}{c}175 \mathrm{FCFA} / \mathrm{kg} \\
10 \mathrm{FCFA} / \mathrm{kg} \\
30 \mathrm{FCFA} / \mathrm{kg}\end{array}$ \\
\hline $\begin{array}{c}\text { Zone VI: } \\
\text { Zone des terres de } \\
\text { barre (Calavi, } \\
\text { Allada) }\end{array}$ & $\begin{array}{c}\text { Drèches de sorgho (drêches de } \\
\text { tchoukoutou) } \\
\text { Tourteaux de soja } \\
\text { Son de maïs } \\
\text { Epluchures de manioc; } \\
\text { Epluchures d'igname } \\
\text { Epluchures de patate } \\
\text { Epluchures d'ananas } \\
\text { Pelures de banane } \\
\text { Pelures de papaye; } \\
\text { Pelures d’orange } \\
\text { Pelures de Artocarpus Altilis (Blèfoutou) } \\
\text { Son de niébé } \\
\text { Coques de riz } \\
\text { Son de riz } \\
\text { Cabosses de cacao } \\
\text { Tourteaux de coprah }\end{array}$ & $\begin{array}{l}+++ \\
+++ \\
+++ \\
+++ \\
+++ \\
+++ \\
+++ \\
+++ \\
++ \\
++ \\
+++ \\
++ \\
+++ \\
+++ \\
+++ \\
+++ \\
++ \\
+++\end{array}$ & $\begin{array}{c}+++ \\
+++ \\
++ \\
++ \\
+++ \\
++ \\
+++ \\
++ \\
++ \\
+++ \\
+++ \\
+++ \\
+++ \\
+++ \\
+++ \\
+++ \\
++ \\
+++\end{array}$ & $\begin{array}{c}\text { Oui } \\
\text { Oui } \\
\text { Oui } \\
\text { Oui } \\
\text { Oui } \\
\text { Non } \\
\text { Non } \\
\text { Oui } \\
\text { Non } \\
\text { Non } \\
\text { Non } \\
\text { Non } \\
\text { Oui } \\
\text { Oui } \\
\text { Oui } \\
\text { Oui } \\
\text { Non } \\
\text { Oui }\end{array}$ & $\begin{array}{c}50 \mathrm{FCFA} / \mathrm{kg} \\
100 \mathrm{FCFA} / \mathrm{kg} \\
50 \mathrm{FCFA} / \mathrm{kg} \\
10 \mathrm{FCFA} / \mathrm{kg} \\
- \\
- \\
30 \mathrm{FCFA} / \mathrm{kg} \\
- \\
- \\
- \\
20 \mathrm{FCFA} / \mathrm{kg} \\
25 \mathrm{FCFA} / \mathrm{kg} \\
175 \mathrm{FCFA} / \mathrm{kg} \\
50 \mathrm{FCFA} / \mathrm{kg} \\
- \\
80 \mathrm{FCFA} / \mathrm{kg}\end{array}$ \\
\hline $\begin{array}{l}\text { ZoneVII : } \\
\text { Zone de la } \\
\text { dépression } \\
\text { (Toffo) }\end{array}$ & $\begin{array}{c}\text { Son de niébé } \\
\text { Son de maïs } \\
\text { Epluchure de manioc } \\
\text { Pelure de banane } \\
\text { Pelure d'orange } \\
\text { Pelures de Artocarpus Altilis (Blèfoutou) } \\
\text { Pelures d'ananas } \\
\text { Pelures de papaye } \\
\text { Tourteaux de coprah }\end{array}$ & $\begin{array}{l}+++ \\
++ \\
+++ \\
++ \\
+++ \\
++ \\
+++ \\
++ \\
+++\end{array}$ & $\begin{array}{l}+++ \\
++ \\
+++ \\
++ \\
++ \\
++ \\
+++ \\
++ \\
+++\end{array}$ & $\begin{array}{l}\text { Oui } \\
\text { Oui } \\
\text { Oui } \\
\text { Non } \\
\text { Non } \\
\text { Non } \\
\text { Oui } \\
\text { Non } \\
\text { Oui }\end{array}$ & $\begin{array}{cc}50 & \mathrm{FCFA} / \mathrm{kg} \\
35 & \mathrm{FCFA} / \mathrm{kg} \\
10 \mathrm{FCFA} / \mathrm{kg} \\
- \\
- \\
30 \mathrm{FCFA} / \mathrm{kg} \\
\quad- \\
80 \mathrm{FCFA} / \mathrm{kg}\end{array}$ \\
\hline
\end{tabular}

+++: Très disponibles ; ++: Disponibles ; +: Peu disponibles

\section{Composition chimique des sous-produits inventoriés}

Dans le tableau 2 ont été présentés les différents sous-produits identifiés ainsi que leurs compositions chimiques. Les sous-produits identifiés pouvaient être regroupés en les cinq catégories suivantes : les sousproduits de racines et tubercules; les sous-produits de fruits; les sousproduits de céréales ; les sous-produits de légumineuses et les tourteaux. Les compositions chimiques ont varié très significativement entre les catégories des sous-produits et même au sein de chaque sous-produit $(\mathrm{P}<0,001)$. Avec une valeur de Fisher ( $F=15,4$ pour MAT), les teneurs en matières azotées ont été les plus influencées par la catégorie de sous-produits. Globalement, les tourteaux avaient des teneurs en MAT très élevées (32,0 \%). Dans cette catégorie de sous-produits, le tourteau de palmiste a présenté une plus faible valeur de MAT (19,8 \%) et le tourteau d'arachide la valeur la plus élevée (54,3 \%). En dehors de la cabosse de cacao pour laquelle une valeur exceptionnellement élevée en MAT (32, 0 \%) a été observée, les MAT des autres sous-produits de fruits n'excèdent guère la barre de $8 \%$. La même tendance a été observée au niveau des sous-produits de racines et de tubercules. Dans la catégorie des sous-produits de légumineuses, les teneurs élevées en MAT étaient à rechercher au niveau des téguments d'arachide, du 
son de niébé, des fanes d’arachide et de niébé, avec des valeurs de 20,4, de 17,3, de 15,5 et de 13, $5 \%$ de MAT. Par ailleurs, les sous-produits de céréales, les drêches de sorgho, les sons de riz et de maïs paraissaient intéressants du point de vue de leurs teneurs en MAT avec respectivement, $19,5,17,1$ et $14,8 \%$.

Quant aux constituants pariétaux, les tourteaux contenaient des teneurs faibles en lignocellulose de 46 \% d’ADF. En moyenne, les autres catégories de sous-produits ont présenté des teneurs en ADF supérieures à 50 $\%$ de matière sèche (MS) et ce constituant pouvait atteindre $65 \%$ dans les sous-produits de céréales. Dans cette catégorie de sous-produits, les teneurs particulièrement élevées en lignocellulose ont été enregistrées au niveau des coques de riz et de sorgho, dans les gousses de soja ainsi qu'au niveau des tiges de sorgho avec, respectivement 76,2, 82,7, 77,3 et 89,9 \% d'ADF).Les sous-produits de céréales pré cités ont présenté également des concentrations élevées en cellulose brute (CB). Ainsi, les coques de sorgho et de riz, les tiges de sorgho, les gousses de soja ont dosé respectivement 29, 2, 37,7, 45, 2 et 49,1 \% de CB par rapport à la matière sèche (MS).

Tableau 2. Composition chimique des sous-produits inventoriés (\% MS) (n =140)

\begin{tabular}{|c|c|c|c|c|c|c|c|c|}
\hline $\begin{array}{l}\text { Ressources } \\
\text { alimentaires }\end{array}$ & MS & $\mathrm{CT}$ & MO & MAT & NDF & $\mathrm{ADF}$ & $\mathrm{CB}$ & MG \\
\hline \multicolumn{9}{|l|}{$\begin{array}{l}\text { Sous-produits } \\
\text { racines et } \\
\text { tubercules }\end{array}$} \\
\hline Epluchures igname & $\begin{array}{c}88,99 \pm \\
0,02 c^{1}\end{array}$ & $\begin{array}{c}5,50 \pm 0 \\
27 \mathrm{~b}\end{array}$ & $\begin{array}{c}93,97 \pm 0 \\
, 25 \mathrm{~b}\end{array}$ & $\begin{array}{c}7,99 \pm 0 \\
01 \mathrm{a}\end{array}$ & $\begin{array}{c}75,31 \pm 0 \\
, 31 \mathrm{a}\end{array}$ & $\begin{array}{c}66,19 \pm 0 \\
, 02 \mathrm{a}\end{array}$ & $\begin{array}{c}4,44 \pm 0 \\
15 \mathrm{c}\end{array}$ & $\begin{array}{l}0,32 \pm \\
0,01 c\end{array}$ \\
\hline Epluchures manioc & $\begin{array}{c}92,75 \pm \\
0.05 a\end{array}$ & $\begin{array}{c}5,45 \pm 0 \\
10 \mathrm{~b}\end{array}$ & $\begin{array}{c}94,67 \pm 0 \\
, 02 \mathrm{~b}\end{array}$ & $\begin{array}{c}5,26 \pm 0 \\
08 \mathrm{~b}\end{array}$ & $\begin{array}{c}60,89 \pm 0 \\
, 03 \mathrm{c}\end{array}$ & $\begin{array}{c}49,36 \pm 0 \\
, 66 \mathrm{c}\end{array}$ & $\begin{array}{c}9,96 \pm 0 \\
02 \mathrm{a}\end{array}$ & $\begin{array}{c}1,80 \pm 0 \\
08 a\end{array}$ \\
\hline Epluchures patate & $\begin{array}{c}88,80 \pm 0 \\
15 \mathrm{c}\end{array}$ & $\begin{array}{c}7,56 \pm 0 \\
24 \mathrm{a}\end{array}$ & $\begin{array}{c}92,82 \pm 0 \\
, 14 \mathrm{c}\end{array}$ & $\begin{array}{c}8,04 \pm 0 \\
04 \mathrm{a}\end{array}$ & $\begin{array}{c}53,59 \pm 0 \\
, 25 \mathrm{~d}\end{array}$ & $\begin{array}{c}43,20 \pm 0 \\
, 49 d\end{array}$ & $\begin{array}{c}5,51 \pm 0 \\
02 \text { b }\end{array}$ & $\begin{array}{c}0,87 \pm 0 \\
03 \mathrm{~b}\end{array}$ \\
\hline Garigo* & $\begin{array}{c}90,60 \pm \\
0.07 \mathrm{~b}\end{array}$ & $\begin{array}{c}0,92 \\
\pm 0,02 \text { с }\end{array}$ & $\begin{array}{c}99,25 \pm 0 \\
, 10 \mathrm{a}\end{array}$ & $\begin{array}{c}1,69 \pm 0 . \\
09 \mathrm{c}\end{array}$ & $\begin{array}{c}66,77 \pm \\
0,05 \text { b }\end{array}$ & $\begin{array}{c}57,77 \pm 0 \\
, 025 b\end{array}$ & $\begin{array}{c}2,15 \pm 0 \\
05 \mathrm{~d}\end{array}$ & $\begin{array}{c}0,91 \pm 0 . \\
05 \mathrm{~b}\end{array}$ \\
\hline Moyenne & $\begin{array}{c}90,28 \pm 0 \\
59 \mathrm{~B}\end{array}$ & $\begin{array}{c}4,85 \pm 0 \\
92 \mathrm{C}\end{array}$ & $\begin{array}{c}95,18 \pm 0 \\
, 92 \mathrm{~A}\end{array}$ & $\begin{array}{c}5,74 \pm 0 \\
98 \mathrm{~B}\end{array}$ & $\begin{array}{c}64,14 \pm 3 \\
, 01 \mathrm{~A}\end{array}$ & $\begin{array}{c}54,13 \\
\pm 3,3 \mathrm{AB}\end{array}$ & $\begin{array}{c}5,51 \pm 1 \\
07 \mathrm{C}\end{array}$ & $\begin{array}{c}0,97 \pm 0 \\
20 \mathrm{~B}\end{array}$ \\
\hline Minimum & 88,65 & 0,90 & 92,68 & 1,60 & 53,34 & 42,71 & 2,15 & 0,31 \\
\hline Maximum & 92,80 & 7,80 & 99,35 & 8,08 & 75,63 & 66,22 & 9,98 & 1,88 \\
\hline CV (\%) & 1,87 & 53,58 & 2,74 & 48,29 & 13,27 & 17,14 & 54,98 & 58,13 \\
\hline \multicolumn{9}{|l|}{ Sous-produits fruits } \\
\hline Pelures bananes & $\begin{array}{c}85,99 \pm 0, \\
29 \mathrm{~d} \\
92,89 \pm 0\end{array}$ & $\begin{array}{c}14,87 \pm 0 \\
.02 \mathrm{~b} \\
9,84 \pm 0\end{array}$ & $\begin{array}{c}85,13 \\
\pm 0.04 \mathrm{~d} \\
90,35\end{array}$ & $\begin{array}{c}6,66 \\
\pm 0,05 \mathrm{c} \\
6,87\end{array}$ & $\begin{array}{c}53,29 \\
\pm 0,10 \mathrm{e} \\
63,22\end{array}$ & $\begin{array}{c}46,99 \\
\pm 0,05 \mathrm{e} \\
41,19\end{array}$ & $\begin{array}{c}9,84 \\
\pm 0.21 \mathrm{~d} \\
14,03 \pm\end{array}$ & $\begin{array}{c}4,59 \\
\pm 0.07 \text { a } \\
2,88\end{array}$ \\
\hline Pelures orange & $\begin{array}{c}01 \text { a } \\
91,39 \pm 0\end{array}$ & $\begin{array}{l}001 \mathrm{~d} \\
10,10\end{array}$ & $\begin{array}{c} \pm 0,18 \text { b } \\
89,94\end{array}$ & $\begin{array}{c} \pm 0,02 \text { c } \\
6,39\end{array}$ & $\begin{array}{c} \pm 0,03 \mathrm{~d} \\
62,64\end{array}$ & $\begin{array}{c} \pm 0,04 \mathrm{f} \\
54,96\end{array}$ & $\begin{array}{c}0,05 \text { b } \\
12,17 \pm\end{array}$ & $\begin{array}{c} \pm 0,03 \mathrm{~b} \\
1,44\end{array}$ \\
\hline Pelures ananas & $\begin{array}{c}16 \mathrm{~b} \\
93,11 \pm 0\end{array}$ & $\begin{array}{c} \pm 0,01 \mathrm{c} \\
7,73\end{array}$ & $\begin{array}{c} \pm 0,03 \mathrm{c} \\
92,47\end{array}$ & $\begin{array}{c} \pm 0,16 \mathrm{~d} \\
7,12\end{array}$ & $\begin{array}{c} \pm 0,09 \mathrm{~d} \\
69,43\end{array}$ & $\begin{array}{c} \pm 0,02 \text { c } \\
50,77\end{array}$ & $\begin{array}{c}0,10 \text { c } \\
8,76\end{array}$ & $\begin{array}{c} \pm 0,22 \mathrm{~d} \\
1,99\end{array}$ \\
\hline Pelures blèfoutou & $\begin{array}{c}39 \text { a } \\
91,13 \pm 0\end{array}$ & $\begin{array}{l} \pm 0,12 \text { e } \\
31,30 \pm 0\end{array}$ & $\begin{array}{c} \pm 0,08 \text { a } \\
68,77\end{array}$ & $\begin{array}{c} \pm 0,09 \mathrm{~b} \\
1,77\end{array}$ & $\begin{array}{c} \pm 0,43 \text { с } \\
92,96 \pm 0\end{array}$ & $\begin{array}{l} \pm 0,04 \mathrm{~d} \\
56,19 \pm 0\end{array}$ & $\begin{array}{c} \pm 0,01 \mathrm{e} \\
8,95\end{array}$ & $\begin{array}{l} \pm 0,02 \text { с } \\
3,15 \pm 0\end{array}$ \\
\hline Peau papaye & $\begin{array}{c}03 \mathrm{~b} \\
85,99 \pm\end{array}$ & $\begin{array}{c}, 02 \mathrm{a} \\
7,55 \pm\end{array}$ & $\begin{array}{c} \pm 0,04 \mathrm{e} \\
92,61\end{array}$ & $\begin{array}{c} \pm 0,02 \mathrm{e} \\
31,97\end{array}$ & $\begin{array}{c}, 005 \mathrm{a} \\
91,30 \pm\end{array}$ & $\begin{array}{c}, 02 \mathrm{~b} \\
66,39 \pm 0\end{array}$ & $\begin{array}{l} \pm 0,01 \mathrm{e} \\
38,56 \pm\end{array}$ & $\begin{array}{l}10 \mathrm{~b} \\
0,97\end{array}$ \\
\hline Cabosse cacao & $0,10 \mathrm{c}$ & $0,01 \mathrm{e}$ & $\pm 0,15 \mathrm{a}$ & $\pm 0,01 \mathrm{a}$ & $0,05 \mathrm{~b}$ & ,04 a & 0,03 a & $\pm 0,01 \mathrm{e}$ \\
\hline
\end{tabular}


15,4

\begin{tabular}{ccccccccc} 
& $90,6733 \pm$ & $13,56 \pm 2$ & $86,54 \pm 2$ & $10,13 \pm$ & $72,14 \pm 4$ & $52,75 \pm 2$ & $\pm 3,17 \mathrm{~A}$ & $2,50 \pm 0$, \\
Moyenne & $0,72 \mathrm{~B}$ &, $49 \mathrm{~A}$ &, $51 \mathrm{C}$ & $2,99 \mathrm{~B}$ &, $49 \mathrm{~A}$ &, $3 \mathrm{AB}$ & $\mathrm{B}$ & $36 \mathrm{~B}$ \\
Minimum & 85,97 & 7,54 & 68,73 & 1,75 & 53,27 & 41,15 & 8,75 & 0,96 \\
Maximum & 93,51 & 31,32 & 92,77 & 31,99 & 92,97 & 66,44 & 39,60 & 4,66 \\
CV (\%) & 2,78 & 63,82 & 10,04 & 102,45 & 21,58 & 15,62 & 71,49 & 50,31 \\
\hline Sous-produits de & & & & & & & & \\
céréales & MS & $\mathrm{CT}$ & MO & MAT & NDF & ADF & CB & MG \\
& $93,98 \pm 0$, & 13,70 & 81,87 & 19,50 & 64,74 & 50,90 & 15,15 & 10,13 \\
Drèche sorgho & $035 \mathrm{~b}$ & $\pm 0,03 \mathrm{~b}$ & $\pm 0,09 \mathrm{f}$ & $\pm 0,12 \mathrm{a}$ & $\pm 0,09 \mathrm{~d}$ & $\pm 0,05 \mathrm{f}$ & $\pm 0,01 \mathrm{~d}$ & $\pm 0,08 \mathrm{~b}$ \\
& 92,75 & 5,13 & 94,93 & 14,84 & $65,56 \pm 0$ & 62,61 & 6,70 & 1,60 \\
Son maïs & $\pm 0,015 \mathrm{~d}$ & $\pm 0,03 \mathrm{f}$ & $\pm 0,03 \mathrm{~b}$ & $\pm 0,05 \mathrm{c}$ &, $035 \mathrm{c}$ & $\pm 0,02 \mathrm{~d}$ & $\pm 0,02 \mathrm{f}$ & $\pm 0,02 \mathrm{~d}$ \\
& & & & 4,05 & & & & \\
& 92,61 & 4,49 & 95,65 & $\pm 0,015$ & 87,33 & 82,66 & $29,19 \pm$ & 2,98 \\
Coques sorgho & $\pm 0,075 \mathrm{e}$ & $\pm 0,26 \mathrm{~g}$ & $\pm 0,02 \mathrm{a}$ & $\mathrm{e}$ & $\pm 0,09 \mathrm{~b}$ & $\pm 0,01 \mathrm{~b}$ & $0,00 \mathrm{c}$ & $\pm 0,05 \mathrm{c}$ \\
& & & & 1,89 & & & & \\
& $93,57 \pm 0$, & 24,77 & 75,21 & $\pm 0,025$ & 87,24 & 76,22 & 37,68 & 0,98 \\
Coque riz & $03 \mathrm{c}$ & $\pm 0,04 \mathrm{a}$ & $\pm 0,03 \mathrm{~g}$ & $\mathrm{~g}$ & $\pm 0,05 \mathrm{~b}$ & $\pm 0,02 \mathrm{c}$ & $\pm 0,08 \mathrm{~b}$ & $\pm 0,01 \mathrm{e}$ \\
& & & & & & & & \\
& 94,53 & 6,56 & 93,69 & 3,29 & 91,59 & 89,88 & 45,18 & $\pm 0,025$ \\
Tige sorgho & $\pm 0,015 \mathrm{a}$ & $\pm 0,23 \mathrm{e}$ & $\pm 0,01 \mathrm{c}$ & $\pm 0,15 \mathrm{f}$ & $\pm 0,04 \mathrm{a}$ & $\pm 0,03 \mathrm{a}$ & $\pm 0,02 \mathrm{a}$ & $\mathrm{f}$ \\
& & 7,49 & & & & & & 0,29 \\
Remoulage riz & 90,10 & $\pm 0,035$ & 92,57 & 10,72 & 48,05 & 62,04 & 1,68 & \pm 0.002 \\
& $\pm 0,05 \mathrm{~g}$ & $\mathrm{~d}$ & $\pm 0,01 \mathrm{~d}$ & $\pm 0,04 \mathrm{~d}$ & $\pm 0,01 \mathrm{e}$ & $\pm 0,01 \mathrm{e}$ & $\pm 0,15 \mathrm{~g}$ & $\mathrm{~g}$ \\
Son de riz & 91,49 & 11,52 & 88,55 & 17,14 & 45,51 & 29,10 & 10,11 & 12,80 \\
& $\pm 0,002 \mathrm{f}$ & $\pm 0,01 \mathrm{c}$ & $\pm 0,04 \mathrm{e}$ & $\pm 0,01 \mathrm{~b}$ & $\pm 0,001 \mathrm{f}$ & $\pm 0,01 \mathrm{~g}$ & $\pm 0,14 \mathrm{e}$ & $\pm 0,01 \mathrm{a}$ \\
Moyenne & $92,72 \pm$ & 10,54 & $88,92 \pm 1$ & $10,20 \pm$ & $70,006 \pm$ & $64,774 \pm$ & $20,8 \pm 4$, & $4,206 \pm$ \\
Minimum & $0,39 \mathrm{~A}$ & $\pm 1,8 \mathrm{AB}$ &, $97 \mathrm{BC}$ & $1,84 \mathrm{~B}$ & $4,9 \mathrm{~A}$ & $5,3 \mathrm{~A}$ & $27 \mathrm{AB}$ & $1,30 \mathrm{~B}$ \\
Maximum & 90,10 & 4,33 & 75,18 & 1,87 & 45,50 & 29,09 & 1,67 & 0,28 \\
CV (\%) & 94,55 & 24,82 & 95,67 & 19,60 & 91,63 & 89,91 & 45,20 & 12,81 \\
\hline & 1,58 & 64,83 & 8,31 & 67,76 & 26,23 & 30,63 & 76,77 & 116,35 \\
\hline
\end{tabular}

Sous-produits

légumineuses

Gousse soja

$93,66 \pm \quad 5,6 \pm 0,0 \quad 94,77 \pm 0 \quad 5,90 \pm 0, \quad 89,12 \pm 0 \quad 77,31 \pm 0 \quad 49,11 \pm \quad 0,65 \pm 0$. $\begin{array}{llllllll}0,045 \mathrm{a} & 30 \mathrm{e} & , 05 \mathrm{c} & 015 \mathrm{e} & , 010 \mathrm{a} & , 07 \mathrm{a} & 0,066 \mathrm{a} & 015 \mathrm{f}\end{array}$

Gousse néré

$85,81 \pm 0, \quad 5,80 \pm 0, \quad 94,26 \pm 0 \quad 0,32 \pm 0 . \quad 72,34 \pm 0 \quad 59,32 \pm 0 \quad 21,77 \pm \quad 1,11 \pm 0$, $04 \mathrm{~g} \quad 015 \mathrm{~d} \quad, 05 \mathrm{~d} \quad 008 \mathrm{~g} \quad, 055 \mathrm{c} \quad, 1 \mathrm{~b} \quad 0,05 \mathrm{~d} \quad 024 \mathrm{~d}$

Poudre néré

$88,59 \pm 0, \quad \pm 0,045 \quad 95,15 \pm 0 \quad 4,49 \pm 0 . \quad 33,80 \pm 0 \quad 24,31 \pm 0 \quad 10,82 \pm \quad 0,96 \pm 0$. $\begin{array}{lllllllll}065 \mathrm{e} & \mathrm{f} & , 10 \mathrm{~b} & 06 \mathrm{f} & , 015 \mathrm{~g} & , 02 \mathrm{~g} & 0,04 \mathrm{~g} & 001 \mathrm{e}\end{array}$

$92,07 \pm 0, \quad 3,96 \pm 1 . \quad 96,04 \pm 0 \quad 17,29 \pm \quad 76,96 \pm 0 \quad 53,64 \pm 0 \quad 14,87 \pm \quad 0,97 \pm 0$.

Son de niébé

$040 \mathrm{c} \quad 90 \mathrm{~g} \quad, 015 \mathrm{a} \quad 0,015 \mathrm{~b} \quad, 035 \mathrm{~b} \quad, 01 \mathrm{~d} \quad 0,03 \mathrm{e} \quad 016 \mathrm{e}$

$92,01 \pm 0, \quad 11,66 \pm 0 \quad 88,46 \pm 0 \quad 13,48 \pm \quad 71,44 \pm 0 \quad 56,28 \pm 0 \quad 30,50 \pm \quad 2,15 \pm 0$.

Fane de niébé

$\begin{array}{llllllll}05 \mathrm{~d} & , 015 \mathrm{a} & , 015 \mathrm{~g} & 0,035 \mathrm{~d} & , 04 \mathrm{~d} & , 02 \mathrm{c} & 0,05 \mathrm{~b} & 01 \mathrm{c}\end{array}$

$86,92 \pm 0, \quad 6,57 \pm 0, \quad 93,46 \pm 0 \quad 15,48 \pm \quad 65,63 \pm 0 \quad 43,52 \pm 0 \quad 22,09 \pm \quad 10,45 \pm$

$\begin{array}{lllllllll}\text { Fane arachide } & 025 \mathrm{f} & 025 & , 03 \mathrm{e} & 0,18 \mathrm{c} & , 10 \mathrm{f} & , 05 \mathrm{f} & 0.035 \mathrm{c} & 0.035 \mathrm{~b}\end{array}$

$\begin{array}{lccccccc}\text { Téguments } \quad 92,36 \pm 0 . & 11,19 \pm 0 & 88,81 \pm 0 & 20,42 \pm & 67,27 \pm 0 & 46,54 \pm 3 & 12,98 \pm & 14,14 \pm\end{array}$ arachide

$\begin{array}{lllll}04 \mathrm{~b} & .01 \mathrm{~b} & .038 \mathrm{f} & 0.019 \mathrm{a}\end{array}$

$90,20 \pm 0, \quad 7,11 \pm 0, \quad 93 \pm 0,79 \quad 11,07 \quad 68,08 \pm \quad 51,56 \quad 23,17 \pm \quad 4,35 \pm 1$,

$\begin{array}{lllllllll}\text { Moyenne } & 78 \mathrm{~B} & 78 \mathrm{BC} & \mathrm{AB} & \pm 1,91 \mathrm{~B} & 4,36 \mathrm{~A} & \pm 4,17 \mathrm{~B} & 3,40 \mathrm{~A} & 42 \mathrm{~B}\end{array}$

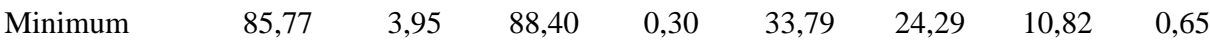

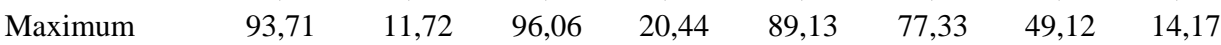

\begin{tabular}{lllllllll} 
CV (\%) & 3,25 & 41,28 & 3,18 & 64,80 & 23,97 & 30,26 & 54,95 & 122,78 \\
\hline
\end{tabular}

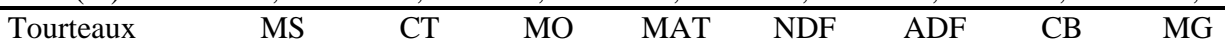

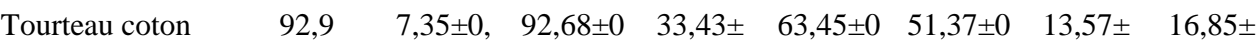




\begin{tabular}{ccccccccc} 
& $\pm 0,01 \mathrm{c}$ & $015 \mathrm{~b}$ &, $02 \mathrm{e}$ & $0,01 \mathrm{c}$ &, $035 \mathrm{e}$ &, $01 \mathrm{c}$ & $0,04 \mathrm{c}$ & $0,01 \mathrm{~d}$ \\
& $93,06 \pm 0$. & $4,45 \pm 0$. & $95,59 \pm 0$ & $19,78 \pm$ & $62,76 \pm 0$ & $43,84 \pm 1$ & $10,49 \pm$ & $22,05 \pm$ \\
Tourteau palmiste & $015 \mathrm{c}$ & $02 \mathrm{f}$ &, $02 \mathrm{a}$ & $0,067 \mathrm{~g}$ &, $39 \mathrm{f}$ & $.02 \mathrm{f}$ & $0.11 \mathrm{f}$ & $0.25 \mathrm{~b}$ \\
& $93,29 \pm 0$, & $7,85 \pm 0$. & $92,22 \pm 0$ & $31,51 \pm$ & 73,58 & $46,13 \pm 0$ & $17,68 \pm$ & $20,72 \pm$ \\
Tourteau soja & $025 \mathrm{c}$ & $11 \mathrm{a}$ &, $045 \mathrm{f}$ & $0,03 \mathrm{~d}$ & $\pm 0,19 \mathrm{a}$ &, $11 \mathrm{e}$ & $0.65 \mathrm{a}$ & $0,08 \mathrm{c}$ \\
& $91,9 \pm 0.4$ & $5,40 \pm 0$. & $94,41 \pm 0$ & $22,94 \pm$ & 65,15 & $58,32 \pm 0$ & $0,96 \pm 0$. & $44,89 \pm$ \\
Tourteau coprah & $2 \mathrm{~d}$ & $08 \mathrm{e}$ &, $21 \mathrm{~b}$ & $0.035 \mathrm{f}$ & $\pm 0,04 \mathrm{~d}$ & $.32 \mathrm{~b}$ & $002 \mathrm{~g}$ & $0,02 \mathrm{a}$ \\
& $95,16 \pm 0$. & $7,78 \pm 0$. & $92,38 \pm 0$ & $27,43 \pm$ & $68,86 \pm 0$ & $60,23 \pm 0$ & $11,17 \pm$ & $14,26 \pm$ \\
Tourteau karité & $085 \mathrm{~b}$ & $050 \mathrm{a}$ & $.11 \mathrm{ef}$ & $0.15 \mathrm{e}$ & $.025 \mathrm{c}$ & $.05 \mathrm{a}$ & $0.07 \mathrm{e}$ & $0.03 \mathrm{f}$ \\
& & 6,11 & & & & & & \\
Tourteau arachide & $90,97 \pm 0$. & \pm 0.001 & $93,94 \pm 0$ & $54,28 \pm$ & $39,67 \pm 0$ & $17,91 \pm 0$ & $16,50 \pm$ & $0,89 \pm 0$. \\
& $40 \mathrm{e}$ & $\mathrm{d}$ & $.001 \mathrm{c}$ & $0.99 \mathrm{a}$ & $.17 \mathrm{~g}$ & $.77 \mathrm{~g}$ & $0.13 \mathrm{~b}$ & $00 \mathrm{~g}$ \\
Soja concacé & $96,12 \pm 0$. & $6,77 \pm 0$. & $93,46 \pm 0$ & $34,81 \pm$ & $72,15 \pm 0$ & $50,45 \pm 0$ & $11,65 \pm$ & $16,26 \pm$ \\
& $075 \mathrm{a}$ & $66 \mathrm{c}$ & $.22 \mathrm{~d}$ & $0.01 \mathrm{~b}$ & $.01 \mathrm{~b}$ & $.01 \mathrm{~d}$ & $0.002 \mathrm{~d}$ & $0,04 \mathrm{e}$ \\
Moyenne & $93,34 \pm 0$, & $6,53 \pm 0$, & $93,52 \pm$ & $32,04 \pm$ & $63,66 \pm 2$ & $46,89 \pm 3$ & $11,71 \pm$ & $19,41 \pm$ \\
Minimum & $45 \mathrm{~A}$ & $32 \mathrm{BC}$ & $0,31 \mathrm{~A}$ & $2,89 \mathrm{~A}$ &, $92 \mathrm{~A}$ &, $61 \mathrm{~B}$ & $1,4 \mathrm{CB}$ & $3,38 \mathrm{~A}$ \\
Maximum & 90,92 & 4,43 & 92,19 & 19,78 & 39,64 & 17,81 & 0,90 & 0,88 \\
CV (\%) & 96,20 & 7,90 & 95,62 & 54,33 & 73,70 & 60,28 & 17,75 & 44,91 \\
& 1,84 & 18,88 & 1,25 & 33,76 & 17,16 & 28,85 & 44,84 & 65,25 \\
Valeur de F & $4,67 * * *$ & $3,83 * * *$ & $3,77 * *$ & $* 3 *$ & $0,57 *$ & $2,27 * *$ & $3,40 * *$ & $* *$ \\
\hline MS Mation
\end{tabular}

MS : Matière sèche ; CT : Cendres totales ; MAT : Matières azotées totales ; CB : Cellulose brute ; NDF :Neutral detergent fiber ; ADF : Acid detergent fiber ; MG : Matières grasses. ${ }^{1}$ Les moyennes suivies de la même lettre dans la même colonne au sein d'une même catégorie de sous-produits (valeur en minuscule) et à travers différentes catégories de sousproduits (valeur en majuscule) ne sont pas significativement différentes $(\mathrm{P}>0,05)$. CV : coefficient de variation. Valeur $\mathrm{F}$ : valeur de Fisher.

${ }^{*}$ Garigo : remoulage manioc. ${ }^{*} \mathrm{p}<0,05 ;{ }^{* *} \mathrm{p}<0,01 ; * * * \mathrm{p}<0,001$.

\section{Valeur nutritive des sous-produits inventoriés}

La digestibilité de la matière organique (dMO) ainsi que les valeurs énergétiques (EB, UFL, UFV) et azotées (MAD, MAD/UFL) des sousproduits ont été présentées dans le tableau 3. En moyenne, les sous-produits de racines et tubercules ainsi que les tourteaux étaient très digestibles et ces valeurs ne descendaient pas en-dessous de 60\% quel que soit le sous-produit considéré. La digestibilité des sous-produits de racines et tubercules étaient de 20 points supérieure à celle des sous-produits de céréales. Les plus faibles valeurs de digestibilité ont été observées au niveau des coques et tiges de céréales ainsi que sur les gousses de légumineuses. Ces digestibilités étaient généralement en dessous de $40 \%$. Les fanes d'arachide sont toujours plus digestibles que les fanes de niébé ( $\mathrm{dMO}=57,8$ contre 49,7\%). La même tendance a été observée au niveau des tourteaux. Les tourteaux de palmiste et de coton étaient moins digestibles que les autres tourteaux. Dans la gamme des sous-produits inventoriés le constater a été que les épluchures de patate, la poudre de néré, les tourteaux (coprah, karité, arachide) avaient des valeurs de digestibilité supérieures à $80 \%$. De fait, de la relation étroite qui a lié la digestibilité des aliments à leurs valeurs énergétiques nettes, les sous- 
produits pré cités avaient des Unités Fourragères Lait (UFL) et des Unités Fourragères Viande (UFV) élevées.

Avec une valeur de $F=30,46$, les matières azotées digestibles (MAD) étaient beaucoup plus influencées par l'effet de catégorie de sousproduits. Bien évidemment tous les tourteaux en contenaient des quantités impressionnantes. Généralement, les sous-produits de céréales et de fruits étaient très pauvres en MAD/UFL, excepté la cabosse de cacao pour laquelle une valeur de 347 g a été observée. En dehors des gousses, tous les autres sous-produits de légumineuses étaient relativement bien pourvus en matières azotées digestibles.

Tableau 3.Valeur nutritive des sous-produits inventoriés ( $\mathrm{n}=140)$

\begin{tabular}{|c|c|c|c|c|c|c|}
\hline $\begin{array}{c}\text { Ressources } \\
\text { alimentaires }\end{array}$ & $\mathrm{dMO}$ & $\mathrm{EB}$ & UFL & UFV & MAD & $\begin{array}{c}\text { MAD/UF } \\
\mathrm{L} \\
\end{array}$ \\
\hline \multicolumn{7}{|l|}{$\begin{array}{l}\text { Sous-produits } \\
\text { racines et tubercules }\end{array}$} \\
\hline Epluchures igname & $\begin{array}{l}77,11 \pm \\
1,37 \quad \mathrm{a}\end{array}$ & $\begin{array}{c}4062 \\
\pm 1,13 \mathrm{c}\end{array}$ & $\begin{array}{c}1,07 \pm \\
0,02 \mathrm{a}\end{array}$ & $\begin{array}{l}1,07 \pm \\
0,03 \mathrm{a}\end{array}$ & $\begin{array}{c}45,77 \pm \\
0,00 \mathrm{a}\end{array}$ & $\begin{array}{c}42,32 \pm \\
0,95 \mathrm{a}\end{array}$ \\
\hline Epluchures manioc & $\begin{array}{c}68,44 \pm \\
4,22 \text { a } \\
81313+\end{array}$ & $\begin{array}{c}4097,28 \pm \\
1,5 b\end{array}$ & $\begin{array}{c}0,93 \pm \\
0,01 \mathrm{a}\end{array}$ & $\begin{array}{l}0,89 \pm \\
0,01 \mathrm{a}\end{array}$ & $\begin{array}{c}20,89 \pm \\
0,52 \mathrm{~b}\end{array}$ & $\begin{array}{c}22,44 \pm \\
1,85 \mathrm{c}\end{array}$ \\
\hline Epluchures patate & $\begin{array}{c}81,313 \pm \\
1,021 \mathrm{a} \\
73,467 \pm\end{array}$ & $\begin{array}{c}4022,5 \pm 2, \\
5 \mathrm{~d} \\
4133,5 \pm\end{array}$ & $\begin{array}{c}1,16 \pm \\
0,00 \mathrm{a} \\
1,01 \pm\end{array}$ & $\begin{array}{l}0,99 \pm \\
0,00 \mathrm{a} \\
0,98 \pm\end{array}$ & $\begin{array}{c}46,166 \pm \\
0,24 \text { a }\end{array}$ & $\begin{array}{c}39, / 1 \pm \\
0,72 \mathrm{~b}\end{array}$ \\
\hline Garigo* & $\underset{74,63 \pm}{a}$ & $\begin{array}{c}3,5 \mathrm{a} \\
4078,8 \pm\end{array}$ & $\begin{array}{l}0,00 \mathrm{a} \\
1,03 \pm\end{array}$ & $\begin{array}{l}0,00 \mathrm{a} \\
0,97 \pm\end{array}$ & $\begin{array}{l}0,00 \quad \text { с } \\
28,18 \pm\end{array}$ & $\begin{array}{l}0,00 \mathrm{~d} \\
26,42 \pm\end{array}$ \\
\hline Moyenne & $1,73 \mathrm{~A}$ & $1,57 \mathrm{BC}$ & $0,00 \mathrm{~A}$ & $0,00 \mathrm{~A}$ & 5,79 A & $5,20 \mathrm{C}$ \\
\hline Minimum & 60,00 & 4020,00 & 0,79 & 0,72 & 0,00 & 0,00 \\
\hline Maximum & 82,50 & 4137,00 & 1,19 & 1,10 & 46,41 & 45,92 \\
\hline CV (\%) & 8,07 & 1,09 & 10,51 & 9,63 & 71,22 & 68,31 \\
\hline \multicolumn{7}{|l|}{ Sous-produits fruits } \\
\hline Pelures bananes & $\begin{array}{c}75,94 \pm \\
3,83 \text { a } \\
74,240 \pm\end{array}$ & $\begin{array}{c}3839,92 \pm \\
0.42 \mathrm{e} \\
4075,85 \pm\end{array}$ & $\begin{array}{l}1,08 \pm \\
0,01 \mathrm{a} \\
1,03 \pm\end{array}$ & $\begin{array}{c}1,03 \pm \\
0,00 \mathrm{a} \\
1,01 \pm\end{array}$ & $\begin{array}{c}33,71 \pm \\
0,58 \mathrm{~d} \\
35,39 \pm\end{array}$ & $\begin{array}{c}31,05 \pm \\
1,76 \mathrm{~b} \\
34,46 \pm\end{array}$ \\
\hline Pelures orange & $\begin{array}{l}3,38 \text { a } \\
66,54 \pm\end{array}$ & $\begin{array}{c}1,35 \mathrm{c} \\
3954,93 \pm\end{array}$ & $\begin{array}{l}0,01 \text { a } \\
0,90 \pm\end{array}$ & $\begin{array}{l}0,01 \mathrm{a} \\
0,87 \pm\end{array}$ & $\begin{array}{c}0,12 \mathrm{c} \\
31,42 \pm\end{array}$ & $\begin{array}{c}2,14 \mathrm{~b} \\
34,09 \pm\end{array}$ \\
\hline Pelures ananas & $\begin{array}{l}0,84 a b \\
73,913 \pm\end{array}$ & $\begin{array}{c}3,60 \mathrm{~d} \\
4082,96 \pm\end{array}$ & $\begin{array}{c}0,02 \mathrm{ab} \\
1,02 \pm\end{array}$ & $\begin{array}{l}0,00 \mathrm{a} \\
1,00 \pm\end{array}$ & $\begin{array}{c}0,97 \mathrm{e} \\
37,95 \pm\end{array}$ & $\begin{array}{c}0,69 \mathrm{~b} \\
36,77 \pm\end{array}$ \\
\hline Pelures blèfoutou & $\begin{array}{c}0,30 \mathrm{a} \\
65,26 \pm\end{array}$ & $\begin{array}{c}0,46 \mathrm{~b} \\
3006,94 \pm\end{array}$ & $\begin{array}{l}0,00 \mathrm{a} \\
0,97 \pm\end{array}$ & $\begin{array}{l}0,00 \mathrm{a} \\
0,91 \pm\end{array}$ & $\begin{array}{c}0,58 \mathrm{~b} \\
0,00\end{array}$ & $\begin{array}{l}0,05 \mathrm{~b} \\
0,00 \pm\end{array}$ \\
\hline Peau papaye & $\begin{array}{l}0,42 a b \\
60,12 \pm\end{array}$ & $\begin{array}{c}0,06 \mathrm{f} \\
4675,65 \pm\end{array}$ & $\begin{array}{l}0,00 \text { a } \\
0,77 \pm\end{array}$ & $\begin{array}{l}0,01 \mathrm{a} \\
0,67 \pm\end{array}$ & $\begin{array}{c} \pm 0,00 \mathrm{~d} \\
265,33\end{array}$ & $\begin{array}{c}0,00 \mathrm{c} \\
347,40 \pm\end{array}$ \\
\hline Cabosse сасао & $\begin{array}{l}4,12 \text { b } \\
69,33 \pm\end{array}$ & $\begin{array}{c}0,65 \mathrm{a} \\
3939,40 \pm\end{array}$ & $\begin{array}{c}0,01 \mathrm{~b} \\
0,96 \pm\end{array}$ & $\begin{array}{l}0,01 \mathrm{~b} \\
0,91 \pm\end{array}$ & $\begin{array}{c} \pm 0,09 \text { a } \\
67,30 \pm 2,9\end{array}$ & $\begin{array}{c}5,98 \mathrm{a} \\
80,63 \pm\end{array}$ \\
\hline Moyenne & $1,67 \mathrm{~A}$ & $1,48 \mathrm{C}$ & $0,00 \mathrm{~A}$ & $0,00 \mathrm{~A}$ & B & $3,32 \mathrm{BC}$ \\
\hline Minimum & 55,45 & 3006,88 & 0,70 & 0,59 & 0,00 & 0,00 \\
\hline Maximum & 83,13 & 4676,30 & 1,21 & 1,09 & 265,43 & 379,88 \\
\hline CV (\%) & 10,251 & 13,08 & 12,92 & 5,31 & 136,81 & 154,3 \\
\hline
\end{tabular}




\begin{tabular}{|c|c|c|c|c|c|c|}
\hline Sous-produits & $\mathrm{dMO}$ & EB & UFL & UFV & MAD & AD/UFL \\
\hline \multirow{3}{*}{ Drèche sorgho } & $76,68 \pm$ & $4761,95 \pm$ & $1,03 \pm 0,0$ & $0,96 \pm$ & $151,36 \pm 0$ & $146,22 \pm$ \\
\hline & 0,71 a & 0,14 a & $1 \mathrm{a}$ & $0,00 \mathrm{a}$ & 58 a & 0,93 b \\
\hline & $68,63 \pm 2,2$ & $4345,05 \pm$ & $0,91 \pm 0,0$ & $0,86 \pm$ & $108,48 \pm 0$ & $118,27 \pm 4$ \\
\hline \multirow[t]{2}{*}{ Son maïs } & $6 \mathrm{a}$ & $0,94 \mathrm{~d}$ & $0 \mathrm{a}$ & $0,00 \mathrm{a}$ & $34 \mathrm{c}$ & $54 \mathrm{c}$ \\
\hline & $31,96 \pm$ & 4423,88 & $0,38 \pm$ & $0,26 \pm$ & $9,59 \pm$ & $25,40 \pm$ \\
\hline \multirow[t]{2}{*}{ Coques sorgho } & $1,84 \mathrm{c}$ & $\pm 0,08 \mathrm{~b}$ & $0,00 \mathrm{c}$ & $0,00 \mathrm{c}$ & 0,009 e & $1,74 \mathrm{e}$ \\
\hline & $36,23 \pm$ & 3452,95 & $0,46 \pm$ & $0,38 \pm$ & $00,00 \pm$ & $0,00 \pm$ \\
\hline \multirow[t]{2}{*}{ Coque riz } & 5,94 с & $\pm 0,05 \mathrm{~g}$ & $0,01 \mathrm{c}$ & $0,0 \mathrm{c}$ & $0,00 \mathrm{f}$ & $0,00 \mathrm{f}$ \\
\hline & $39,86 \pm 2,7$ & 4396,26 & $0,48 \pm 0,0$ & $0,37 \pm 0,0$ & $0,00 \pm 0,00$ & $0,00 \pm 0,00$ \\
\hline \multirow[t]{2}{*}{ Tige sorgho } & $3 c$ & $\pm 0,26 \mathrm{c}$ & $0 \mathrm{c}$ & $0 \mathrm{c}$ & $\mathrm{f}$ & $\mathrm{f}$ \\
\hline & $70,40 \pm$ & 3989,14 & $0,97 \pm 0,0$ & $0,88 \pm 0,0$ & $70,62 \pm 0,0$ & $73,33 \pm$ \\
\hline \multirow[t]{2}{*}{ Remoulage riz } & $3,68 \mathrm{a}$ & $\pm 0,14 \mathrm{f}$ & $1 \mathrm{a}$ & $0 \mathrm{a}$ & $63 \mathrm{~d}$ & $4,76 \mathrm{~d}$ \\
\hline & $53,35 \pm$ & $4320,84 \pm$ & $0,68 \pm$ & $0,59 \pm 0,0$ & 129,43 & $191,48 \pm$ \\
\hline \multirow[t]{2}{*}{ Son de riz } & 3,27 b & $0,09 \mathrm{e}$ & $0,05 \mathrm{~b}$ & $6 \mathrm{~b}$ & $\pm 0,060 \mathrm{~b}$ & $4,15 \mathrm{a}$ \\
\hline & $53,87 \pm$ & $4241,4 \pm 1$, & $0,70 \pm$ & $0,61 \pm$ & $67,07 \pm$ & $79,24 \pm$ \\
\hline Moyenne & $3,92 \mathrm{~B}$ & $60 \mathrm{BC}$ & $0,01 \mathrm{~B}$ & $0,01 \mathrm{~B}$ & $3,36 \quad \mathrm{~B}$ & $5,71 \mathrm{BC}$ \\
\hline Minimum & 28,40 & 3452,90 & 0,33 & 0,21 & 0,00 & 0,00 \\
\hline Maximum & 77,85 & 4763,41 & 1,11 & 0,99 & 151,94 & 205,81 \\
\hline CV (\%) & 33,34 & 9,38 & 37,86 & 45,8 & 91,34 & 90,9 \\
\hline \multicolumn{7}{|l|}{$\begin{array}{l}\text { Sous-produits } \\
\text { légumineuses }\end{array}$} \\
\hline \multirow{3}{*}{ Gousse soja } & $49,33 \pm 1,4$ & $4461 \pm 3.2$ & $0,61 \pm$ & $0,51 \pm 0,0$ & $26,44 \pm$ & $43,13 \pm$ \\
\hline & $4 \mathrm{~d}$ & $7 \mathrm{~d}$ & $0,0018 \mathrm{~d}$ & $021 \mathrm{~b}$ & $0,61 \mathrm{e}$ & $1,33 \mathrm{~d}$ \\
\hline & $35,86 \pm$ & $4136 \pm 1.3$ & $0,43 \pm 0,0$ & $0,32 \pm$ & $0,00 \pm 0,00$ & $0,00 \pm 0,00$ \\
\hline \multirow[t]{2}{*}{ Gousse néré } & $0,21 \mathrm{e}$ & $7 \mathrm{f}$ & 033 е & 0,0033 с & g & $\mathrm{f}$ \\
\hline & $84,97 \pm 0,1$ & $4128,5 \pm 0$. & $1,21 \pm$ & $1,11 \pm 0,0$ & $15,36 \pm$ & $12,65 \pm$ \\
\hline \multirow[t]{2}{*}{ Poudre néré } & $9 \mathrm{a}$ & $63 \mathrm{~g}$ & 0,0066 a & 053 a & $0,91 \mathrm{f}$ & $0,51 \mathrm{e}$ \\
\hline & $78,49 \pm 0,5$ & $4473,75 \pm$ & $1,07 \pm 0,0$ & $1,04 \pm$ & $130,92 \pm$ & 121,17 \\
\hline \multirow[t]{2}{*}{ Son de niébé } & $5 \mathrm{~b}$ & $2.61 \mathrm{c}$ & $01 \mathrm{~b}$ & 0,001 a & $0,21 \mathrm{~b}$ & $\pm 1,26$ с \\
\hline & 49,68 & $4255,56 \pm$ & $0,63 \pm$ & $0,53 \pm$ & $89,90 \pm 0,2$ & $144,29 \pm$ \\
\hline \multirow[t]{2}{*}{ Fane de niébé } & $\pm 2,23 \mathrm{~d}$ & $2.74 \mathrm{e}$ & $0,003 \mathrm{~d}$ & $0,0041 \mathrm{~b}$ & $4 \mathrm{~d}$ & 4,26 b \\
\hline & $57,84 \pm$ & $4863,63 \pm$ & $0,73 \pm$ & $0,62 \pm$ & $109,16 \pm$ & $147,43 \pm$ \\
\hline \multirow[t]{2}{*}{ Fane d'arachide } & 0,55 с & $2.64 \mathrm{a}$ & $0,0010 \mathrm{c}$ & $0,0010 \mathrm{~b}$ & 1,13 с & $2,05 \mathrm{~b}$ \\
\hline & $51,030 \pm$ & $4797 \pm 0.3$ & $0,63 \pm$ & $0,51 \pm 0,0$ & $159,38 \pm 0$ & $247,023 \pm$ \\
\hline \multirow[t]{2}{*}{ Téguments arachide } & $0,61 \mathrm{~d}$ & $2 \mathrm{~b}$ & $0,0088 \mathrm{~d}$ & 088 b & 12 a & 0,33 a \\
\hline & $58,17 \pm$ & 4445,06 & $0,76 \pm$ & $0,66 \pm$ & $75,88 \pm$ & $102,24 \pm$ \\
\hline Moyenne & $3,63 \mathrm{~B}$ & $\begin{array}{c}\text { B } \\
4136 \pm 1.3\end{array}$ & $0,0058 \mathrm{~B}$ & $0,0061 \mathrm{~B}$ & $12,86 \mathrm{~B}$ & 7,88 B \\
\hline Minimum & 35,79 & $\begin{array}{c}7 \\
4461 \pm 3.2\end{array}$ & 0,43 & 0,32 & 0,00 & 0,00 \\
\hline \multirow{2}{*}{$\begin{array}{c}\text { Maximum } \\
\text { CV (\%) }\end{array}$} & 85,32 & 7 & 1,23 & 1,18 & 159,63 & 407,97 \\
\hline & 28,16 & 6,43 & 34,91 & 42,53 & 77,69 & 83,93 \\
\hline \multirow{3}{*}{ Tourteaux } & & & & & & MAD/UF \\
\hline & $\mathrm{dMO}$ & EB & UFL & UFV & MAD & L \\
\hline & $67,28 \pm 0,1$ & $5305,54 \pm$ & $0,87 \pm 0,0$ & $0,78 \pm$ & $278,74 \pm 0$ & $318,64 \pm 1$, \\
\hline \multirow[t]{2}{*}{ Tourteau coton } & $4 \mathrm{~b}$ & $0,21 \mathrm{c}$ & $066 \mathrm{~b}$ & $0,01 \mathrm{bc}$ & $06 \mathrm{c}$ & $94 \mathrm{~b}$ \\
\hline & $60,82 \pm 0,2$ & $4983,37 \pm$ & $0,77 \pm 0,0$ & $0,67 \pm$ & $153,88 \pm 0$ & $198,60 \pm 0$ \\
\hline Tourteau palmiste & $6 \mathrm{c}$ & $0,37 \mathrm{f}$ & 033 с & $0,00 \mathrm{c}$ & $15 \mathrm{~g}$ & $56 \mathrm{~d}$ \\
\hline
\end{tabular}




\begin{tabular}{ccccccc} 
& $71,63 \pm 1,3$ & $5378,16 \pm$ & $0,94 \pm 0,0$ & $0,86 \pm$ & $263,41 \pm 1$, & $278,87 \pm 7$, \\
Tourteau soja & $7 \mathrm{~b}$ & $0,16 \mathrm{~b}$ & $02 \mathrm{~b}$ & $0,03 \mathrm{~b}$ & $19 \mathrm{~d}$ & $36 \mathrm{c}$ \\
& $87,47 \pm 3,1$ & $6707,41 \pm$ & $1,20 \pm 0,0$ & $1,17 \pm$ & $182,10 \pm 0$, & $151,66 \pm 6$, \\
Tourteau coprah & $9 \mathrm{a}$ & $0,09 \mathrm{a}$ & $052 \mathrm{a}$ & $0,06 \mathrm{a}$ & $21 \mathrm{f}$ & $50 \mathrm{e}$ \\
& $84,26 \pm 3,1$ & $5041,40 \pm$ & $1,15 \pm 0,0$ & $1,11 \pm$ & $222,31 \pm 0$, & $193,99 \pm$ \\
Tourteau karité & $9 \mathrm{a}$ & $0.40 \mathrm{e}$ & $5 \mathrm{a}$ & $0,01 \mathrm{a}$ & $67 \mathrm{e}$ & $8,45 \mathrm{~d}$ \\
& $82,63 \pm 3,1$ & $4660,26 \pm$ & $1,12 \pm 0,0$ & $1,08 \pm$ & $469,90 \pm 0$, & $419,77 \pm 8$, \\
Tourteau arachide & $9 \mathrm{a}$ & $0.32 \mathrm{~g}$ & $52 \mathrm{a}$ & $0,06 \mathrm{a}$ & $15 \mathrm{a}$ & $77 \mathrm{a}$ \\
& $80,19 \pm$ & $5267,29 \pm$ & $1,08 \pm 0,0$ & $1,02 \pm$ & $291,38 \pm$ & $269,14 \pm 1$, \\
Soja concacé & $0,40 \mathrm{a}$ & $0,29 \mathrm{~d}$ & $033 \mathrm{a}$ & $0,01 \mathrm{a}$ & $0,060 \mathrm{~b}$ & $14 \mathrm{c}$ \\
& $76,32 \pm$ & $5334,8 \pm 1$, & $1,02 \pm$ & $0,96 \pm$ & $265,96 \pm$ & $261,53 \pm$ \\
Moyenne & $2,15 \mathrm{~A}$ & $67 \mathrm{~A}$ & $0,0033 \mathrm{~A}$ & $0,00 \mathrm{~A}$ & $21,36 \mathrm{~A}$ & $21,36 \mathrm{~A}$ \\
Minimum & 60,31 & 4659,50 & 0,77 & 0,67 & 153,58 & 138,94 \\
Maximum & 93,69 & 6707,50 & 1,31 & 1,30 & 470,06 & 444,90 \\
CV (\%) & 12,95 & 11,75 & 15,78 & 19,54 & 36,81 & 33,21 \\
& & & & & & \\
Valeur de F & $10,88 * * *$ & $15,84 * * *$ & $10,29 * * *$ & $10,96 * * *$ & $30,46 * * *$ & $15,22 * * *$ \\
\hline
\end{tabular}

dMO : digestibilité de la matière organique (en \%) ; EB : énergie brute (kcal/kg MS) ; UFL

et UFV : Unité fourragère lait et viande (/kg MS) ; MAD : Matières azotées digestibles (g/kg de MS). ${ }^{1}$ Les moyennes suivies de la même lettre dans la même colonne au sein d'une même catégorie de sous-produits (valeur en minuscule) et à travers différentes catégories de

sous-produits (valeur en majuscule) ne sont pas significativement différentes $(\mathrm{p}>0,05)$.

$\mathrm{CV}$ : coefficient de variation. Valeur $\mathrm{F}$ : valeur de Fisher. *Garigo : remoulage manioc. * ${ }^{*}<$ 0,$05 ; * * \mathrm{p}<0,01 ; * * * \mathrm{p}<0,001$.

\section{Discussion}

Dans les zones agro écologiques investiguées, l'agriculture et la transformation des produits agricoles constituent les activités économiques majeures des populations. D’après les statistiques de la Direction de l'Elevage, les quantités stockées des résidus agricoles avoisinent 11. 043. $369 \mathrm{~kg}$ (DE, 2012). Au nombre des sous-produits agricoles stockés, les fanes de légumineuses 3.303.831, les tiges de maïs 3.051.204 et de riz 4464662 kg viennent en tête. Même s'il n'existe pas encore d'utilisation à l'échelle industrielle de ces sous-produits agricoles, ils constituent un appoint de grande importance dans la couverture des besoins du bétail pendant la saison sèche où les graminées fourragères et les autres herbacées deviennent très rares. Dans les zones où les racines et tubercules sont bien développés (notamment, les zones vivrières du Sud-Borgou, cotonnières du CentreBénin et des terres de barre), les agroéleveurs les intègrent de plus en plus fortement dans le processus d'embouche ou de complémentation en période de déficit alimentaire. La valeur marchande des fanes de légumineuses est toujours plus élevée que celles des tiges de céréales et des épluchures de racines et tubercules. Elles prennent de plus en plus de valeur au fur et à mesure que l'on monte dans la zone Nord-Soudanienne du Bénin caractérisée par une saison de pluie et une saison sèche. 
Il n'existe pas de données quantitatives sur les sous-produits de transformation artisanale de céréales (son de maïs et drêche de sorgho). Toutefois, ces sous-produits sont disponibles dans toutes les zones agroécologiques du Bénin et leur valeur marchande est toujours plus faible dans la zone de production des cultures vivrières du Sud-Borgou que les autres zones. D’après les statistiques rendues disponibles par la FAO en 2014 sur la disponibilité des résidus et sous-produits agro-industriels dans l'espace UEMOA, 1,89 et 0,62 tonnes par UBT sont respectivement produites pour les épluchures de manioc et d'igname (FAO, 2014). Par rapport aux sousproduits agro-industriels, 46.887 ; 32.811 et 52.105 tonnes de tourteaux de coton, de soja et d'arachide sont respectivement disponibles par UBT. En dehors de rares cas de rupture de stock des sources d'approvisionnement et de pénurie saisonnière des sous-produits agricoles et autres résidus de récoltes liés à la saisonnalité de la production, les données sus indiquées montrent assez bien que les sous-produits sont disponibles à tout moment.

En déterminant les valeurs nutritionnelles des ressources alimentaires localement disponibles au Bénin, Toléba et al. (2004) se sont intéressés à six (6) sous-produits : spathes de maïs, fanes d'arachide, coques de niébé, farine de manioc, épluchure de manioc et son de maïs. Cinq années plus tard, ces auteurs ont élargi la base de données en s'intéressant aux valeurs nutritionnelles de l'ananas (couronnes entières, lamelles de couronne et pelures) de tourteaux (soja, palmiste, arachide et coton) de son de riz et de patate douce (Toléba et al., 2009). Les résultats sus indiqués montrent assez bien le caractère disparate des informations disponibles sur les sous-produits agricoles et sous-produits agro industriels du Bénin. Ces études sont réalisées dans deux zones agro écologiques du Bénin (zone des pêcheries, et des terres de barre) et permettent d'évaluer les caractéristiques nutritionnelles de 15 sous-produits agricoles et de transformation artisanale. L'étude relative à 5 zones agro écologiques (zone cotonnière du Nord-Bénin, zone vivrière du Sud-Borgou, zone cotonnière du Centre Bénin, zone des terres de barre et zone de dépression) permet d'inventorier et de déterminer les valeurs nutritives de 31 sous-produits agricoles et sous-produits agro industriels.

De point de vue de la détermination des valeurs nutritives de ces sous-produits, l'étude n'est pas encore complète par rapport à celle réalisée dans plusieurs travaux (INRA, 1988 ; Sauvant et al., 2004 ; INRA, 2007). La liste des sous-produits agricoles et agro industriels inventoriés n'est certainement pas encore exhaustive, car l'étude a embrassé cinq zones agro écologiques sur les huit que compte le Bénin. En outre, il reste à déterminer les teneurs en minéraux ( $\mathrm{P}$ et $\mathrm{Ca}$ ), ainsi que les teneurs en amidon et en acides aminés (lysine et méthionine). Toutefois, pour l'instant, on peut se satisfaire des informations fournies par ce travail afin d'assurer une 
complémentation alimentaire adéquate aux animaux pendant la saison déficitaire.

Au Bénin, les sous-produits de fruits recensés dans ce travail ont des teneurs MAT en-dessous de 8\%. Ainsi comme le soulignent plusieurs nutritionnistes (Milford et Minson, 1966 ; Minson, 1990 ; Coleman et al., 2003), il faut un minimum de teneur en MAT de 8\% de MS pour assurer aux microorganismes du rumen un fonctionnement adéquat. En dehors de la cabosse de cacao pour laquelle une valeur exceptionnellement élevée des MAT (32, 0\%) est observée, les teneurs en MAT des autres sous-produits de fruits sont limitantes pour le bon fonctionnement du rumen. En condition tropicale humide du Bénin, il a été rapporté sur l'engraissement des moutons Djallonké qu'il faut une quantité en énergie nette de 0,60 UFL et des valeurs azotées de $90 \mathrm{~g}$ MAD/UFL pour obtenir des GMQ de $50 \mathrm{~g} / \mathrm{j}$ chez ces animaux (Babatoundé et al. (2008). Les sous-produits de racines et tubercules, de fruits et de céréales ont tous des énergies nettes supérieures à cette limite et sont de ce fait, de véritables pourvoyeurs d'énergies nettes pour les rations alimentaires destinées à ces animaux. Au ? des teneurs en matières azotées, tous les sous-produits pré cités ne donnent pas satisfaction. Dans la gamme des sous-produits inventoriés il est intéressant de constater que les sous-produits de légumineuses et les tourteaux en contiennent des quantités très élevées et peuvent être insérés avantageusement dans les plans d’alimentation des ruminants.

\section{Conclusion}

L’étude permet d’inventorier et de déterminer les disponibilités, les valeurs marchandes et nutritionnelles des sous-produits agricoles et agroindustriels des zones agro écologiques du Bénin. Les tiges de céréales, les fanes de légumineuses sont disponibles et plus utilisés dans l'alimentation des ruminants. Ces sous-produits prennent de la valeur au fur et à mesure que l'on évolue vers les zones du Nord-Bénin. Parmi les sous-produits de transformation, les sons de céréales et les tourteaux sont les plus utilisés. Les tourteaux de soja et de coton sont plus disponibles que les tourteaux de palmiste, de karité et de coprah. La valeur marchande des tourteaux est toujours plus élevée que celle des autres sous-produits. Au regard des valeurs nutritives des sous-produits agricoles et agroindustriels, les résultats obtenus offrent des possibilités de choix de compléments alimentaires aux agroéleveurs pour un engraissement des ruminants en milieu tropical humide du Bénin. Pendant la saison sèche où les graminées et les autres herbacées fourragères deviennent rares dans les parcours naturels, ils peuvent corriger le déséquilibre quantitatif et qualitatif des rations destinées aux ruminants en utilisant un ou plusieurs sous-produits. Si les éleveurs décident d'utiliser deux types de compléments, l'un pour la couverture des besoins énergétiques 
et l'autre pour les besoins azotés, ils peuvent porter leurs choix sur les sousproduits de céréales, de racines et tubercules ou de fruits en ce qui concerne l’énergie et les fanes de légumineuses pour les besoins azotés. Si par contre, ces éleveurs décident de n’utiliser qu'un seul concentré pour la couverture des besoins énergétiques et azotés, ce sont les tourteaux qui répondent à cette exigence. L’étude offre aussi la possibilité aux opérateurs économiques qui désirent s’investir dans le domaine de l'élevage des ruminants de monter leur projet en ayant en même temps dans un seul document les informations sur les disponibilités saisonnières, les valeurs nutritives et marchandes des différents sous-produits agricoles et agroindustriels susceptibles d'être valorisés au Bénin.

\section{Remerciements :}

Les auteurs remercient infiniment la Banque Mondiale à travers le projet "Alim’Bloc" du Programme de Productivité Agricole en Afrique de l’Ouest (PPAAO) pour avoir financé cette étude.

\section{References:}

1. AOAC (2000). Officiel Methods of Analysis,17th ed., AOAC Int, Washington, DC,USA.

2. Babatounde S. (2005). Etude et prediction de la valeur alimentaire de graminées et de légumineuses fourragères en zone tropicale humide du Bénin (thèse de doctorat), Gembloux, Faculté universitaires des sciences agronomiques, $268 \mathrm{p}$.

3. Babatounde S., Toleba S. S., Adandedjan C.C., Dahouda M., Sidi H., Buldgen A. (2008) Comportement alimentaire et évolution pondérale des moutons djallonke sur des pâturages de fourrages cultivés en mélange. Annales des Sciences Agronomiques du Bénin 10 (1) : 3149.

4. CIRAD (2009). Drêche ensilée, tourteau de palmiste et coques de cacao Digestibilité comparée des rations contenant de la drêche ensilée des brasseries, du tourteau de palmiste ou des coques de cacao chez le porc en croissance finition au Cameroun déterminer la digestibilité des rations contenant des taux croissants de la drêche ensilée des brasseries, du tourteau de palmiste et des coques de cacao chez le porc en croissance finition. http://pigtrop.cirad.fr

5. Coleman S.W., Hart S.P., Sahlu T. (2003). Relationships among forage chemistry, rumination and retention time with intake and digestibility of hay by goats. Small Ruminant Research. 50 : 129-140.

6. Dagnelie P. (1986). Théorie et méthodes statistiques. Applications agronomiques, vol. 2. Les

7. presses agronomiques de Gembloux, A.S.B.L. (Belgique), 463 p. 
8. DE (Direction de L’Elevage du Bénin), 2012. Annuaire statistique. $85 \mathrm{p}$.

9. Jarrige R., et Demarquilly C. (1981). Prévision de la valeur nutritive des aliments des ruminants Ed INRA Publications, Versailles 40 p.

10. FAO, 2014 : Résidus agricoles et sous-produits agro-industriels en Afrique de l'ouest :Rapport sur l' Etat des lieux et perspectives pour l’élevage. 73p.

11. France J, Dijkstra J, Dhanoa MS, Lopez S and Bannik A : 2000. Estimating the extent of degradation of ruminant feeds from a description of their gas production profiles observed in vitro : derivation of models and other mathematical considerations. $\mathrm{Br}$. $J$ Nutr 83 : 143 - 150 .

12. INRA (1988). Alimentation des bovins, ovins et caprins. Jarrige R. (ed). INRA publications, Paris, $471 \mathrm{p}$.

13. INRA (2007). Alimentation des bovins, ovins et caprins : Besoins des animaux-Valeurs des

14. aliments, Tables Inra 2007, 307p.

15. INSAE (2012). Statistiques/Statistiques économiques/ Produit intérieur brut (http://www.insae-bj.org/produit-interieur.html).

16. INSAE (2015). Grands traits sur le commerce extérieur au Bénin : Rapport sur les échanges

17. extérieures du Bénin en 2014, 25p.

18. Jarrige R. (1981). Les constituants glucidiques des fourrages: variations, digestibilité et dosage. In : Prévision de la valeur nutritive des aliments des ruminants, (ed). INRA publ., versailles France, pp 13-40.

19. Menke KH, Raab L, Salewski A, Steingass H, Fritz D and Schneider W : 1979. The estimation of the digestibility and metabolizable energy content of ruminant feeding stuffs from the gas production when they are incubated with rumen liquor in vitro. J. Agric.

20. Sci.Camb. 93 : 217-222.

21. Menke K.H., Steingass H. (1988). Estimation of the energetic value obtained from chemical analysis and in vitro gas production using rumen fluid. Anim. Res. Dev. 28 : 7-55.

22. Milford R., Minson D. J. (1966). Intake of tropical pasture species. Proceedings of the IX International Grasslands Congress 1: 815 822.

23. Milford R., Minson D. J. (1966). Intake of tropical pasture species. Proceeding of the International Grasslands Congress 1: 815 - 822.

24. Minson D.J. (1990). The chemical composition and nutritive value of tropical grasses $\mathrm{In}$ : 
25. Skerman P. J., Riveros F (eds), Tropical grasses. FAO plant production and protection series.

26. 23, Food and Agriculture Organization

27. Rivière R. (1991). Manuel de l'alimentation des ruminants en milieu tropical. Paris, France :

28. la Documentation Française, 527 p.

29. Sauvant D., Perez J.-M., Tran G. (eds), (2004). Tables de composition et de valeur nutritive des matières premières destinées aux animaux d'élevage : porcs , volailles, bovins, ovins,

30. Caprins, lapins,chevaux,poissons. INRA Editions et AFZ, Paris. 301p.

31. Sinsin B. (1993). Phytosociologie, écologie, valeur pastorale, production et capacité de charge des pâturages naturels du périmètre Nikki-Kalalé au Nord-Bénin. Thèse de Doctorat en sciences agronomiques. Université de Bruxelles, Belgique 390p.

32. Toléba, S.S., Adandédjan C., Babatoundé, S., Dahouda M., Bahini D. (2004). Identification et détermination de la valeur des ressources fourragères disponibles pour l'alimentation des petits ruminants dans le Sud-Ouest du Bénin. In : Revue Africaine de Santé et de Production Animale, 2 (3-4) : 213-222.

33. Toléba S.S., Youssao A. K. I., Dahouda M., Missainhoun U. M. A., Mensah G.A. (2009) Identification et valeurs nutritionnelles des aliments utilisés en élevage d'aulacodes (Thryonomysswinderianus) dans les villes de Cotonou et Porto-Novo au Bénin. Bulletin de la Recherche Agronomique du Bénin, Numéro 64 - Juin 2009. 